\title{
Thermal Transient Measurements of an Ultra-Low-Power MOX Sensor
}

\author{
F. Rastrello, ${ }^{1}$ P. Placidi, ${ }^{1}$ L. Abbati, ${ }^{1}$ A. Scorzoni, ${ }^{1}$ E. Cozzani, ${ }^{2}$ I. Elmi, ${ }^{2}$ \\ S. Zampolli, ${ }^{2}$ and G. C. Cardinali ${ }^{2}$ \\ ${ }^{1}$ Dipartimento di Ingegneria Elettronica e dell'Informazione, Università degli Studi di Perugia, Via G. Duranti, \\ 9306125 Perugia, Italy \\ ${ }^{2}$ CNR-IMM, Via P. Gobetti 101, 40129 Bologna, Italy
}

Correspondence should be addressed to P. Placidi, pisana.placidi@diei.unipg.it

Received 15 July 2010; Accepted 21 September 2010

Academic Editor: P. Siciliano

Copyright ( ) 2010 F. Rastrello et al. This is an open access article distributed under the Creative Commons Attribution License, which permits unrestricted use, distribution, and reproduction in any medium, provided the original work is properly cited.

\begin{abstract}
This paper describes a system for the simultaneous dynamic control and thermal characterization of the heating of an Ultra Low Power (ULP) micromachined sensor. A Pulse Width Modulated (PWM) powering system has been realized using a microcontroller to characterize the thermal behavior of a device. Objectives of the research were to analyze the relation between the time period and duty cycle of the PWM signal and the operating temperature of such ULP micromachined systems, to observe the thermal time constants of the device during the heating phase and to measure the total thermal conductance. Constant target heater resistance experiments highlighted that an approximately constant heater temperature at regime can only be obtained if the time period of the heating signal is smaller than $50 \mu \mathrm{s}$. Constant power experiments show quantitatively a thermal time constant $\tau$ that decreases during heating in a range from $2.3 \mathrm{~ms}$ to $2 \mathrm{~ms}$ as a function of an increasing temperature rise $\Delta T$ between the ambient and the operating temperature. Moreover, we calculated the total thermal conductance. Finally, repeatability of experimental results was assessed by guaranteeing the standard deviation of the controlled temperature which was within $\pm 5.5^{\circ} \mathrm{C}$ in worst case conditions.
\end{abstract}

\section{Introduction}

Gas sensors made with semiconducting metal oxides (MOX) are devices based on thermally assisted chemical reactions between their sensing surface and the species to be detected [1-3]. For best sensitivity and selectivity, the devices are kept at a temperature ranging from $200^{\circ} \mathrm{C}$ to $450^{\circ} \mathrm{C}$ generally through an integrated heating resisto based on $\mathrm{Pt}$ or poly-Si.

Usually a silicon micromachined MOX sensor consists of an electrically conductive suspended structure over a thin, solid, and nonconducting membrane. The conductive part can be divided in two elements, one is dedicated to control the operating temperature, the heating part and the other one is a semiconducting metaloxide layer, the sensitive part. The heater can receive a controlling signal to drive the sensitive part of the sensor to the desired operating temperature, while the sensitive part changes its conductive behavior in presence of specific oxidizing or reducing gases. This variation can be measured with other conductive contacts connected to the sensing material.

In order to enhance sensor selectivity and to minimize the overall gas sensor power consumption an ON/OFF heating signal control is recommended (pulsed mode operation) [4]. Thus, for optimal temperature control an important requirement is a good comprehension of the transient temperature behavior of the system, under relatively fast applied voltage pulses, and its time constant. It is essential to know how a pulsed signal changes the temperature of the heater and of the overall microsystem, relatively to its time period and duty cycle. Moreover, when used in this discontinuous temperature mode, a good understanding of the hotplate thermal dynamics is of uppermost importance, in order to efficiently design ultra-low-power driving electronics for low-cost applications, as proposed in [5].

In the literature we can find different types of MOX gas sensors featuring integrated heating resistors [1-7], controlled by a number of interface acquisition systems [8-10], 


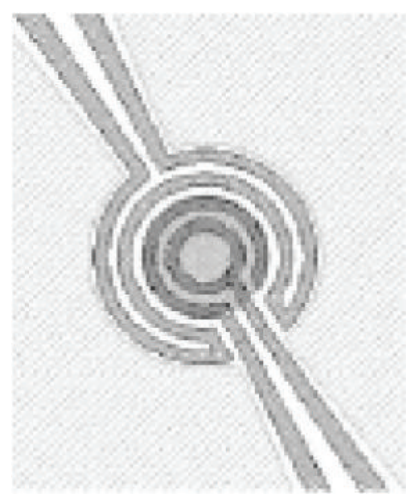

(a)

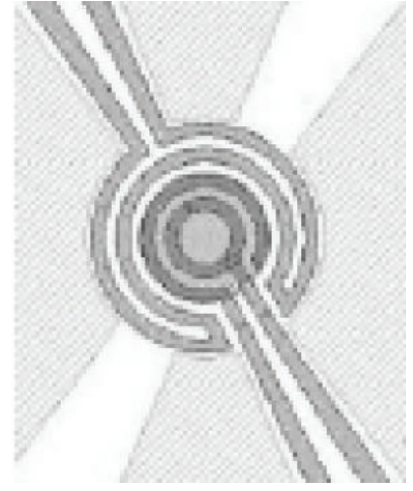

(b)

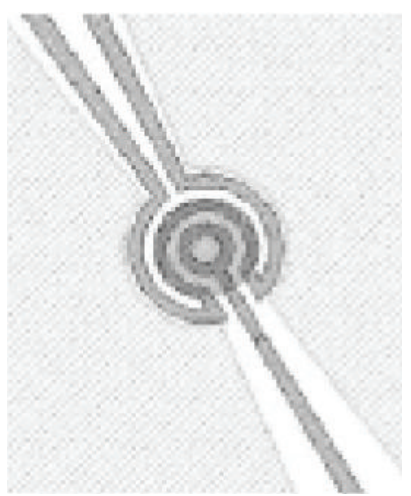

(e)

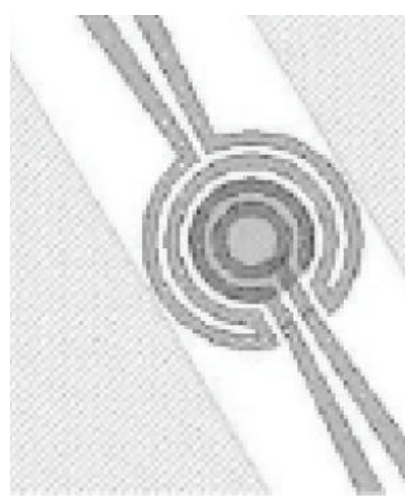

(c)

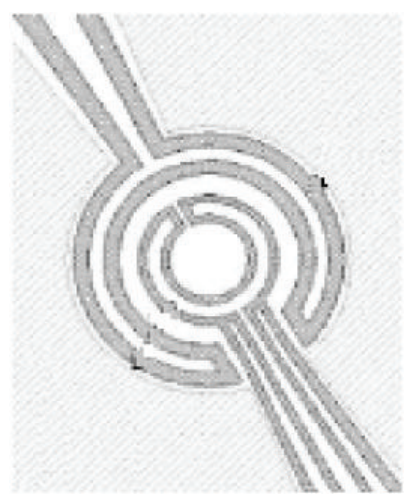

(f)

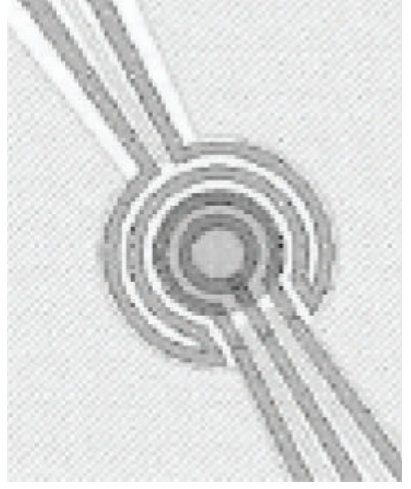

(d)

FIGURE 1: ULP hotplates with different heaters, contacts, and suspending arms layouts $[16,17]$.

whereas there is not much about thermal characterization. In fact they are thermally studied only with single voltage pulses in order to calculate their thermal constant, such as in [11-15]. Instead, a simple embedded system suitable to thermally characterize a device using PWM signals featuring a microcontrolled tunable pulse width could be very useful in order to gather a better comprehension of MOX sensor thermal transient behavior.

In this paper a thorough dynamic thermal characterization of a micromachined Pt temperature sensor surrounded by a Pt heating resistor and sitting on a silicon-based membrane is presented. The device belongs to a family of innovative gas sensors (Figure 1) dubbed Ultra-Low-Power (ULP) gas sensors, where the heating part (microheater) and sensing part (temperature sensor or $T$-sensor) are extremely miniaturized and interlocked $[10,16,17]$. The thermal characterization is carried out using an embedded system, reusable for the temperature control of other similar ULP MOX gas sensors.

The microheater is used to set the sensor operating temperature $\left(T_{S}\right)$. The thermal transient characterization is performed by applying to the heater a PWM square voltage waveform with variable duty cycle and frequency generated by an ARM7TDMI-S microcontroller $(\mu \mathrm{C})$, combined with a simple conditioning circuit, suitable for controlling the heating power and measuring the relative heater resistance values. The integrated Pt $T$-sensor has been used to calibrate the resistance of the heating resistor. The resistance of the $T$ sensor has been acquired by means of a digital oscilloscope using a constant current supply and an amplification module.

In the remainder of the paper we introduce the device under test (Section 2) and the experimental setup (Section 3). Then we present in Section 4 the thermal characterization of the device and the study to assess the measurement repeatability. The conclusions are given in Section 5 .

\section{ULP Device}

The ULP MOX sensors were proposed for emerging ultralow-power applications like wireless sensor networks and semipassive RFID tags for food logistics applications [5]. The overall power consumption of the ULP hotplates is reduced by scaling the dimensions of the device features to the limits achievable with low-cost contact lithography and wet silicon bulk micromachining technology. In order to reach a good temperature uniformity on the active area of the sensors, a circular geometry with concentric heaters and electrodes was chosen and thoroughly optimized through FEM simulations [16].

The ultra-low-power sensor under test is a suspended structure created with silicon bulk micromachining techniques. It consists of a non-conducting membrane, patterned 
by reactive ion etching, which is a support and thermal insulating bridge for conductive platinum strips, realized by selective etching.

In ULP MOX sensors of the same family, the dielectric membrane provides optimum thermal isolation between the substrate and the gas-sensitive heated area. The latter will also be indicated as "active area". Numerical simulation results, confirmed by thermographic analysis [16], showed that, when fed with constant power, in this area the temperature is constant within a maximum error of about $10 \%$ (due to localized hot and cold spots) when measured in ${ }^{\circ} \mathrm{C}$.

This particular device is formed by a 2-contact ( 5 and 6 in Figure 4) Pt horseshoe heater with tapered arms and a 4-contact Pt resistor, that is, the T-sensor, patterned with the same platinum layer. FEM simulations showed that the thermal behavior observed in this experiment have generic validity for other similar ULP devices such as those described in [16].

To be used as hotplates for ULP MOX gas sensors, the 4 -contact $T$-sensor is replaced by 2 contact electrodes as shown in Figures 1(a)-1(e). These electrodes are used to measure the conductance of a metal-oxide-semiconductor thin film sensing layer, typically consisting of $60-80 \mathrm{~nm}$ of tin oxide deposited by a modified rhetoaxial growth and thermal oxidation (M-RGTO) technique.

A SEM micrograph of the sensing layer is shown in Figure 2. Noble metal catalyst nanoclusters are selectively deposited on some sensors of the array to enhance sensitivity and selectivity and are visible as bright white spots on the SEM micrograph.

Figure 3(a) shows two arrays of ULP MOX sensors bonded on a TO-8 case, on top of the low-volume test chamber used for their characterization, while Figure 3(b) shows an optical micrograph of a 4-sensor array, together with the $25 \mu \mathrm{m}$ diameter Al/Si wedge-bonding wires.

Details on the ULP MOX sensors fabrication process with M-RGTO deposited sensing layers and their performance as acquired with laboratory electronics can be found in [17].

By using the sputtering techniques with mechanical shadow masks reported in [17], particular attention was devoted to avoid the use of photoresist or solvents which would result in sensing layer contamination. However, with this masking technique, it is difficult to deposit different materials within the four independent hotplates of the $1.5 \mathrm{~mm}$ wide ULP array chip. For this reason, a microdropcoating technique was successfully proposed, as described in [6]. By using the drop-coating technique, different materials can be deposited as single microdrops on the different hotplates within a single array, enabling the production of four heterogeneous and independent ULP MOX sensors within a single $1.5 \times 1.0 \mathrm{~mm}^{2}$ chip, to be used in enose configuration with pattern recognition techniques for qualitative analyses of complex mixtures.

\section{Experimental Setup}

For electrical acquisitions we exploited an experimental setup based on the electronic system with the aim of measuring the thermal transient behavior of the whole

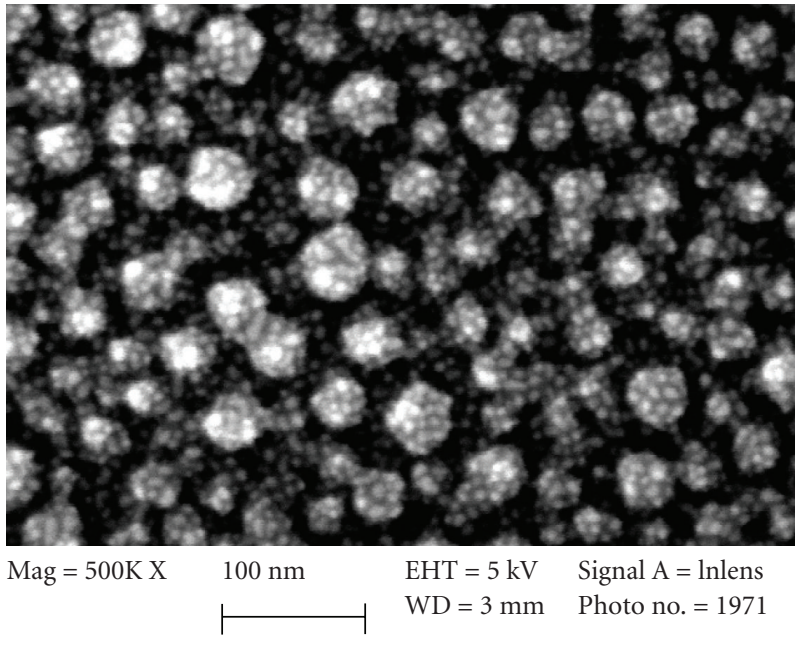

Figure 2: SEM micrograph of a M-RGTO deposited $\mathrm{SnO}_{2}$ film with Au catalyst nanoclusters.

structure by implementing a PWM powering signal, used to keep as low as possible the power dissipation in the driving circuit. Figure 4 shows the block diagram of the measurement system used to evaluate the transient response of the device under test.

The measurement system of Figure 4 is essentially composed of (i) an ARM7TDMI-S based $\mu \mathrm{C}$, embedded in a development board, used to generate the PWM heating voltage and to measure the heater resistance; (ii) a Keithley $2400 \mathrm{SMU}$, to give constant current $I_{T \text {-sens }}=60 \mu \mathrm{A}$ to the $T$-sensor and at the same time to measure its own output voltage (2-wire measurement, useful just to check over sensor integrity); (iii) a BB 128p INA, to amplify the output voltage of the $T$-sensor that we use to calculate the temperature and analyze the transient; (iv) an Agilent 54621A oscilloscope, used to plot the T-sensor output voltage; (v) a currentvoltage converter, included in the conditioning circuit and based on a low noise, high bandwidth-gain product OPA228P operational amplifier, used to measure the heating current.

The $\mu \mathrm{C}$ has been programmed to generate a customizable PWM (obtained by summing two PWM signals, thanks to an ad hoc conditioning circuit), with selectable period $\left(\tau_{\mathrm{PWM}}\right)$ and duty cycle: a heating interval $\left(\tau_{\text {hot }}\right)$ to heat the active area, an acquisition interval ( $\tau_{\text {acq }}$ of 64 internal $\mu \mathrm{C}$ peripheral clock counts, about $4 \mu \mathrm{s}$ ) to make electrical acquisitions on the heater resistor with the ARM, and a cooling interval $\left(\tau_{\text {cool }}\right)$ in which no power is dissipated. When computing the delivered power, the $\mu \mathrm{C}$ takes into account both $\tau_{\text {hot }}$ and $\tau_{\text {acq }}$ time intervals.

Two different control methods were implemented, namely, constant target heater resistance and constant heating power, as described in the remainder of the paper.

\section{Thermal Characterization}

4.1. Thermal Analysis: Heater and T-Sensor Calibration. The resistance versus temperature behavior of the $T$-sensor was 


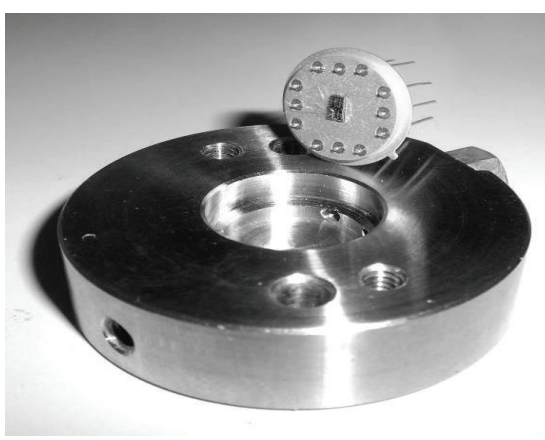

(a)

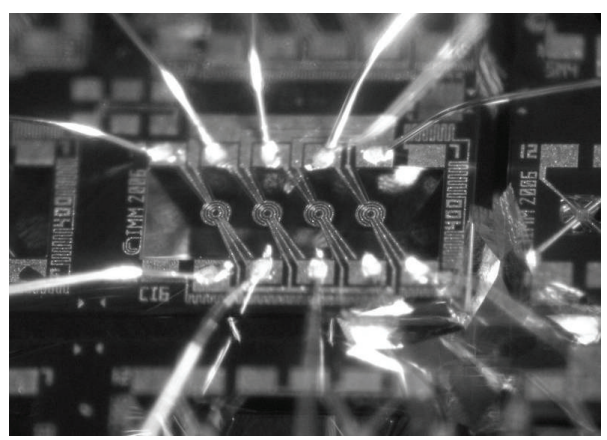

(b)

FIGURE 3: ULP sensor arrays bonded on a TO-8 case on top of the chamber used for its characterization (a) and optical micrograph of a wire-bonded sensor array (b).

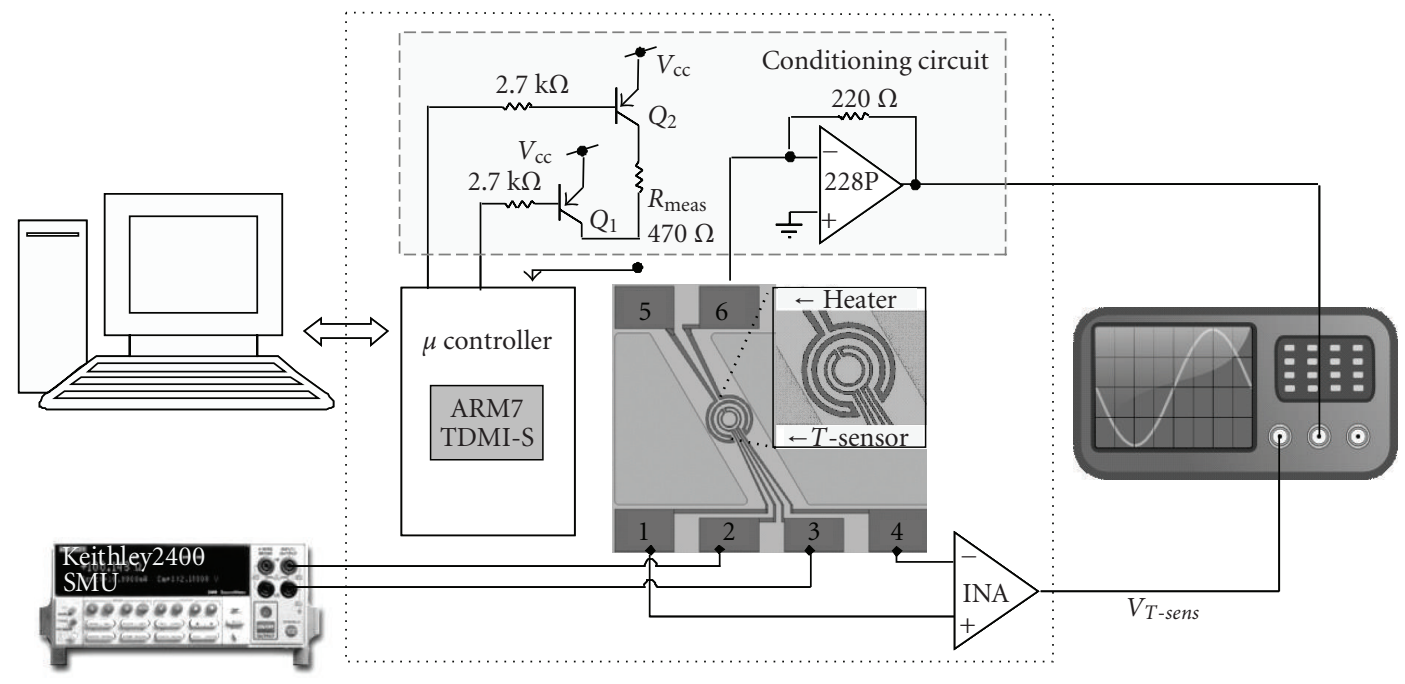

FIGURE 4: Block diagram of the experimental setup for device transient characterization.

first DC-characterized at the wafer level using a temperaturecontrolled thermochuck and standard laboratory instrumentation, and its temperature coefficient of resistance (TCR) was extracted, as described in [16], exploiting the typical temperature-dependent first-order function for the resistance of metals, that is,

$$
R_{S}\left(T_{S}\right)=R_{S 0}\left(1+\mathrm{TCR}_{0} \cdot T_{S}\right),
$$

where $R_{S}$ and $R_{S 0}$ are the $T$-sensor resistances at temperatures $T_{S}$ and $T_{0}=0^{\circ} \mathrm{C}$, respectively, and $\mathrm{TCR}_{0}$ is its temperature coefficient of resistance at $0{ }^{\circ} \mathrm{C}: \mathrm{TCR}_{0}=0.00305 \mathrm{~K}^{-1}$. The sensor temperature can be extracted from (1), by means of a 4-wire measurement of $R_{S}\left(T_{S}\right)$. In fact, applying a small current $\left(I_{\mathrm{T} \text {-sens }}=60 \mu \mathrm{A}\right)$ to the contacts $\# 2$ and \#3 and acquiring the voltage at the contacts \#1 and \#4 (Figure 4), after the amplification module, we measure $V_{T \text {-sens }}$, from which we obtain $R_{S}\left(T_{S}\right): R_{S}\left(T_{S}\right)=V_{T \text {-sens }} /\left(I_{T \text {-sens }} \cdot G_{\mathrm{INA}}\right)$, where $G_{\text {INA }}$ is the INA amplifying gain. From (1) we calculate the T-sensor temperature as.

$$
T_{S}=\left(\frac{R_{S}\left(T_{S}\right)}{R_{S 0}}-1\right) \cdot \frac{1}{\mathrm{TCR}_{0}} .
$$

About the calibration of the heater resistance $R_{H}$, a dynamic thermal characterization was accomplished applying a $20 \mathrm{kHz}$ PWM signal to reach the operating resistance. This is the maximum frequency that allowed us to keep pace with different functions of the $\mu \mathrm{C}$ algorithm: power management, data acquisition, dynamic duty cycle calculation, and so forth While keeping the substrate at ambient temperature $T_{a}$, the $\mu \mathrm{C}$ measured the heater resistance $R_{H}$ and the regime active area temperature was measured through the $T$-sensor. Supposing that the regime temperature of the heater $T_{H}$ is exactly identical to the regime $T_{S}$ measured on the $T$-sensor, we obtained a $R_{H}=R_{H}\left(T_{H}\right)$ calibration curve. At the same time a simple, physically based, linear analytical model of the resistance of the heater as a function of the temperature was developed as follows:

$$
R_{H}=R_{H 0}\left\lfloor 1+\mathrm{TCR}_{0} T_{a}+\mathrm{TCR}_{\text {eff }}\left(T_{H}-T_{a}\right)\right\rfloor,
$$

where $R_{H 0}$ is the heater resistance at $0^{\circ} \mathrm{C}$ and TCR $\mathrm{Teff}_{\text {is }}$ an effective TCR which in general is different from the $T$-sensor value $\mathrm{TCR}_{0}$ and takes into account the geometrical features of the heater [18]. It was experimentally verified that (3) fits 
very well the experimental measurements with the following parameters: $R_{H 0}=114.2 \Omega, \mathrm{TCR}_{\mathrm{eff}}=0.00252 \mathrm{~K}^{-1}, T_{a}=27^{\circ} \mathrm{C}$.

4.2. Thermal Transients during Heating: Constant Target Heater Resistance. By exploiting the inverse relationship of (3), that is, $T_{H}=T_{H}\left(R_{H}\right)$, a routine was implemented in the $\mu \mathrm{C}$ firmware, able to dynamically calculate the duty cycle $(\mathrm{DC})=\tau_{\text {hot }} / \tau_{\mathrm{PWM}}$, at constant PWM period $\tau_{\mathrm{PWM}}$ in order to reach a given heater resistance or, identically, heater temperature.

The algorithm, based on fuzzy logic, tries to minimize the time needed to reach the target resistance value. Using PWMs of different periods $(50,200,1000,5000 \mu \mathrm{s})$ and choosing several target heater resistances $R_{H}$ (or $T_{H}$ values), we observed the heating transients of the heater, measured in the time domain when the substrate was at ambient temperature $T_{a}=27^{\circ} \mathrm{C}$. This was accomplished using the current-voltage converter concisely described in Section 3. heating is accomplished at constant voltage; Therefore, the heater resistance is simply calculated by dividing this constant voltage by the measured current.

Referring to Figures $5(\mathrm{a})$ to $5(\mathrm{~d})$, when applying PWM signals to reach a target $R_{H}$ of $200 \Omega$ (corresponding to $T_{H}$ of about $300^{\circ} \mathrm{C}$ ), the stationary waveform of $R_{H}$ depends on DC and the signal period $\tau_{\mathrm{PWM}}$. This stationary behavior can be an approximately constant value at regime only if the heating signal is much faster than the time constant of the device (Section 4.3). In particular we observed that with a heating period of $50 \mu \mathrm{s}$, after a transient time which is not displayed in the figure, a stable heating resistance $R_{H}$ is not reached (Figure 5(a)). In fact we highlighted a ripple of resistance of about $5 \Omega$, that corresponds to a temperature ripple of about $15^{\circ} \mathrm{C}$. Please note that in all the thermal characterizations described in Sections 4.3 and 4.4 this ripple is not shown because the measurements are taken with the microcontroller instead of the current-voltage converter: the microcontroller samples the heater resistance only at the end of the $\tau_{\text {hot }}$ time interval, that is, once every $50 \mu \mathrm{s}$, which is the present resolution limit of our measurement setup. Moreover, in Figure 5(a) we can note a ringing in the first part of the heating period due to the influence of parasitic capacitances on the current-voltage converter. A simple SPICE simulation confirmed these experimental results. The temperature ripple of Figure 3(a) is not detectable on the $T$-sensor due to its relatively high noise, as described in Section 4.4 .

When increasing $\tau_{\mathrm{PWM}}$ at $200 \mu$ s while keeping the same peak level for $R_{H}$, a bigger ripple appears at regime for $R_{H}$, as highlighted in Figure 5(b). In this case we also observe a significant temperature ripple on the $T$-sensor. This ripple, both on $R_{H}$ and $T_{S}$, worsens with increasing target operating resistance. Finally, with longer PWM periods of $1 \mathrm{~ms}$ (Figure 5(c)) and $5 \mathrm{~ms}$ (Figure 5(d)) we observe everincreasing resistance and temperature ripples.

4.3. Thermal Transients: Constant Heating Power. This part of the thermal characterization was focused on constant heating power to extract the total thermal conductance $G_{\text {th }}$ of the device. A second objective of this characterization was to compare the transient behavior and time constants of the heater and the $T$-sensor in the heating phase.

A specific purpose code was implemented on the ARM7 $\mu \mathrm{C}$ to feed the heater with a constant electric power with the PWM signal. The heater resistance was measured during $\tau_{\text {acq }}$ (see Section 3 ) by exploiting the voltage divider made of $R_{\text {meas }}$ and the heater resistance, as shown in Figure 4 . The power delivered to the heater during measurement (about $0.45 \mathrm{~mW}$ in the worst case of maximum operative resistance, i.e., $R_{H}=230 \Omega$ ) was included in the overall calculated power.

Previous studies [12] referred to a not-ULP device without passivation layer have shown that the dependence of the T-sensor temperature $T_{S}$ on the dissipated power of the active area $\left(P_{\mathrm{aa}}\right)$, is a non linear relationship and can be expressed as follows:

$$
P_{\mathrm{aa}}=G_{\mathrm{th}, \mathrm{eff}}\left(T_{S}\right) \cdot\left(T_{S}-T_{a}\right),
$$

where $G_{\text {th,eff }}\left(T_{S}\right)(\mathrm{W} / \mathrm{K})$ is an effective thermal conductance, function of the temperature, which accounts for the conduction, convection, and radiation heat transfer mechanisms. However, in our test structure it is not possible to measure the power dissipated in the active area only, instead we are able to measure the total power $P_{\text {tot }}$ dissipated on the heater, including the power in the current carrying taps.

Therefore, we supposed to deal with a new effective thermal conductance:

$$
G_{\mathrm{th}, \mathrm{eff}}^{\prime}=\frac{P_{\mathrm{tot}}}{\left(T_{S}-T_{a}\right)},
$$

and the expression for $G_{\text {th,eff }}^{\prime}$ has been proposed in [12]:

$$
G_{\mathrm{th}, \mathrm{eff}}^{\prime}=\left(G_{\mathrm{th}, a}+B\right)+A \cdot\left(T_{S}-T_{a}\right)-\frac{B \Delta T_{t}}{\left[T_{S}-T_{a}+\Delta T_{t}\right]},
$$

where $G_{\mathrm{th}, a}(\mathrm{~W} / \mathrm{K})$, accounts for the thermal conductance at ambient temperature, $A\left(\mathrm{~W} / \mathrm{K}^{2}\right), B(\mathrm{~W} / \mathrm{K})$, and $\Delta T_{t}(\mathrm{~K})$ are suitable constants [12].

Figure 6(a) shows the total power $P_{\text {tot }}$ as a function of $T_{S}$. The nonlinear behavior of this graph clearly indicates that the effective thermal conductance of (6) is not constant as a function of the active area temperature $T_{S}$. Figure $6(\mathrm{~b})$ shows this effective thermal conductance $G_{\text {th,eff }}^{\prime}$ as a function of the heating power $\left(P_{\text {tot }}\right)$, together with a best-fit line based on (5). It must be underlined that the experimental points at low temperature are not enough for reliably extracting the value of the parameter $G_{\mathrm{th}, a}$. Therefore, $G_{\mathrm{th}, a}$ was supposed as a known parameter during the fitting procedure $\left(G_{\mathrm{th}, a}=\right.$ $\left.1.0 \times 10^{-5} \mathrm{~W} / \mathrm{K}\right)$, while more reliable values for $\left(G_{\mathrm{th}, a}+B\right)$ and $A$ were calculated, obtaining that $\left(G_{\mathrm{th}, a}+B\right)=(3 \pm$ $0.02) \times 10^{-5} \mathrm{~W} / \mathrm{K}$ and $A=(2.51 \pm 0.08) \times 10^{-8} \mathrm{~W} / \mathrm{K}^{2}$, respectively. In the operating temperatures $T_{S}-T_{a}$ from 100 to $400^{\circ} \mathrm{C}$ (i.e., (2-15) $\mathrm{mW}$ ), the thermal conductance can also be approximated by a linear function, shown as a black line in Figure 6(b), with

$$
G_{\text {th,eff }}=2.913 \times 10^{-5}+2.638 \times 10^{-8} \cdot\left(T_{S}-T_{a}\right)(\mathrm{W} / \mathrm{K}) .
$$




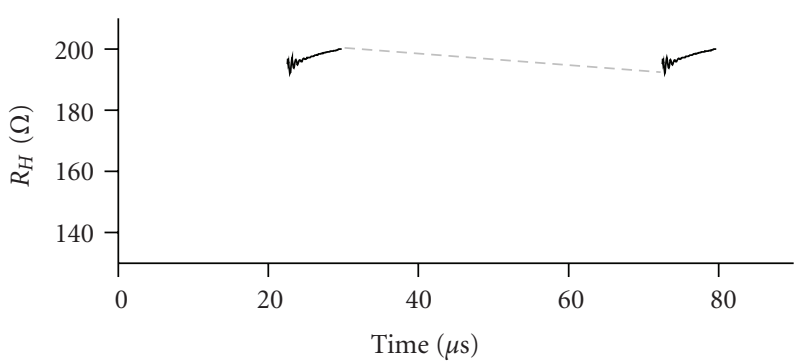

(a)

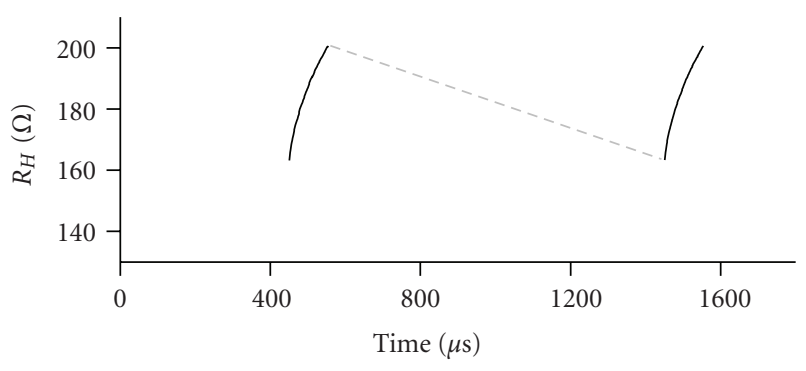

(c)

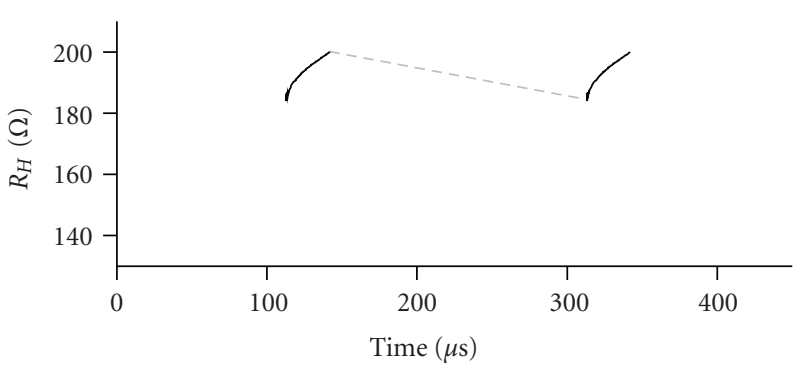

(b)

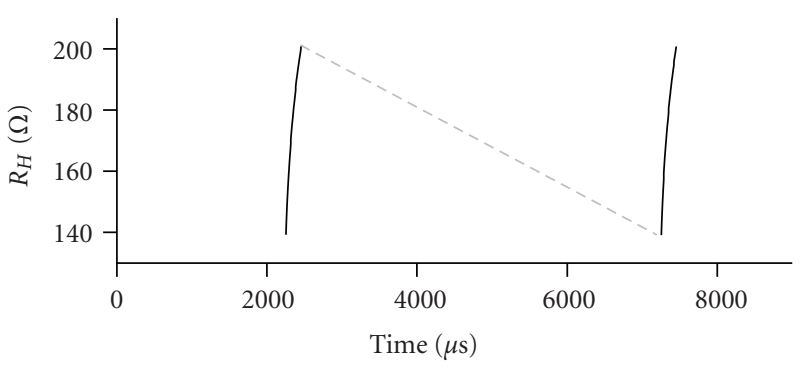

(d)

FIGURE 5: Heating phases at regime: measured responses obtained applying a periodic PWM stimulus with different $\tau_{\text {PWM }}$ periods. In each heating cycle, heating was stopped when a target value $R_{H}=200 \Omega$ was reached. Ambient temperature $T_{a}=27^{\circ} \mathrm{C}$. Heating pulse period $\tau_{\mathrm{PwM}}$ $=50 \mu \mathrm{s}(\mathrm{a}), \tau_{\mathrm{PWM}}=200 \mu \mathrm{s}(\mathrm{b}), \tau_{\mathrm{PWM}}=1 \mathrm{~ms}(\mathrm{c})$, and $\tau_{\mathrm{PWM}}=5 \mathrm{~ms}(\mathrm{~d})$. Dashed lines show an imaginary and rectified cooling transient that was not directly measured.

About the thermal transients, we carried out constant power measurements of the temperature transients at constant ambient temperature $\left(T_{a}=27^{\circ} \mathrm{C}\right)$ during heating, both on the heater (task accomplished by the $\mu \mathrm{C}$ ) and the $T$ sensor (through the INA and the oscilloscope as described in Section 3). A PWM period of $50 \mu$ s was used in order to minimize unwanted temperature ripples with the present version of the $\mu \mathrm{C}$ firmware. The experimental results of the transient behavior are shown in Figure 7.

By fitting experimental data with a single exponential we observed a time constant $\tau$ identical for both the heater and $T$-sensor, which decreases with increasing applied power, that is, with increasing the temperature gap $\Delta T$ between the regime temperature and the ambient temperature $T_{a}$, in a range that starts from about $2.3 \mathrm{~ms}$ at constant heating power of $1 \mathrm{~mW}$ (i.e., $\Delta T \approx 60^{\circ} \mathrm{C}-T_{a}$ ) to about $2 \mathrm{~ms}$ at constant heating power of $14 \mathrm{~mW}$ (i.e., $\Delta T \approx 390^{\circ} \mathrm{C}-T_{a}$ ). This result is explained by an increasing thermal conductance as a function of the increasing temperature (see again Figure 6): should the overall thermal capacitance $C_{\text {th }}$ be approximately constant, then the thermal time constant $C_{\text {th }} / G_{\text {th,eff }}$ will decrease for increasing temperature.

4.4. Measurement Repeatability. To qualify and quantify the repeatability of the measurement system we made a study on the standard deviation $(\sigma)$ of the acquired temperature values for 5 different thermal transients (Table 1). For temperature measurements performed during the heating phase on the $T$-sensor the $\sigma_{T \text {-sens }}$ grows with the power applied (i.e., $\Delta T$ ): $\sigma_{T \text {-sens }}$ starts from $3.5^{\circ} \mathrm{C}$ when the regime temperature is $60^{\circ} \mathrm{C}$ and grows up to $5.5^{\circ} \mathrm{C}$ when the regime
TABle 1: Measurement standard deviation of the heater and $T$ sensor at different power conditions.

\begin{tabular}{lcc}
\hline$P_{\text {tot }}$ (heating) $(\mathrm{mW})$ & $\sigma_{H}\left({ }^{\circ} \mathrm{C}\right)$ & $\sigma_{T \text {-sens }}\left({ }^{\circ} \mathrm{C}\right)$ \\
\hline 1 & 1 & 3.5 \\
7 & 1.2 & 4 \\
14 & 1.2 & 5 \\
\hline
\end{tabular}

temperature is about $450^{\circ} \mathrm{C}$ (i.e., $\max P_{\text {tot }} \approx 15 \mathrm{~mW}$ ). An increasing behavior can be found for the standard deviation $\sigma_{H}$ of the temperature measurements performed on the heater: $\sigma_{H}$ starts from $1^{\circ} \mathrm{C}$ when the regime temperature is $60^{\circ} \mathrm{C}$ and grows up to $1.5^{\circ} \mathrm{C}$ when the regime temperature is about $450^{\circ} \mathrm{C}$. The higher current used when measuring the heater temperature with respect to the low measuring current of the $T$-sensor can explain this lower standard deviation. In fact the electrical noise due to the PWM control is equally present on the heater and the $T$-sensor; however, its effective value must be compared with the measured voltage drop, which is much higher in the case of the heater.

\section{Conclusion}

The sensitivity, selectivity, and response time of semiconducting metal oxide gas sensors strongly depend on the sensing layer temperature, which is usually determined by a heating resistor integrated in the sensor structure. In this context a thermal characterization of a micromachined ULP device has been done using an embedded system with PWM control—a $\mu \mathrm{C}$ unit with an ARM7 core-by exploiting a 


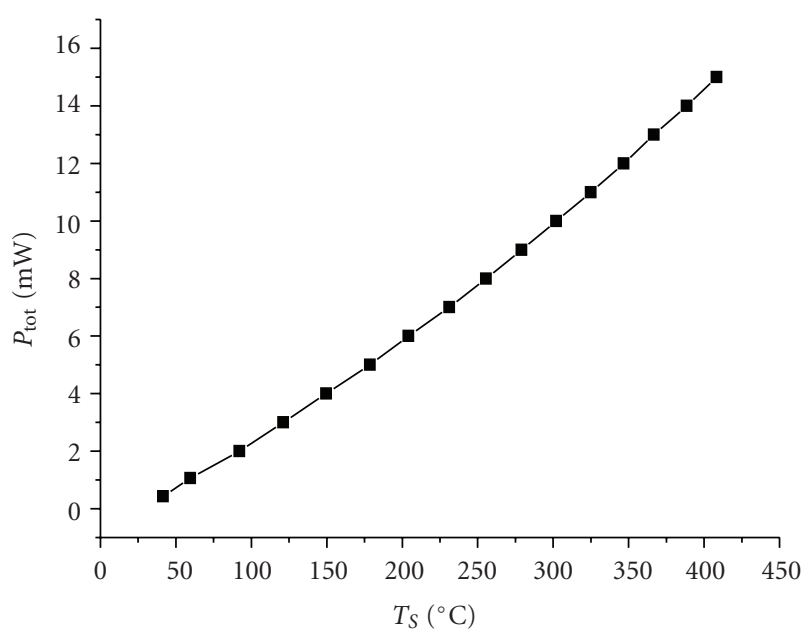

(a)

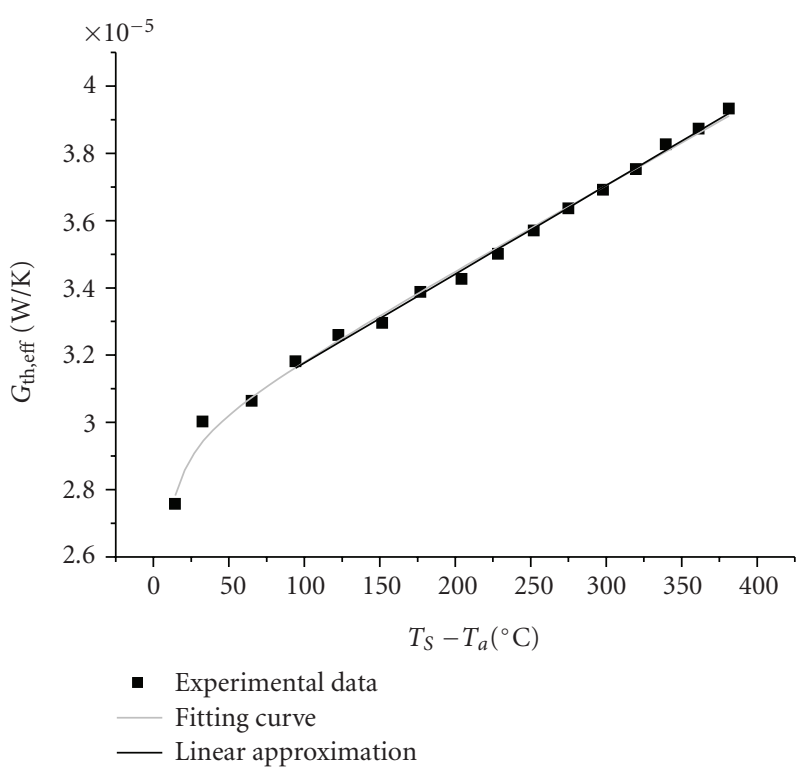

(b)

Figure 6: (a) Total power $\mathbf{P}_{\text {tot }}$ in stationary condition as a function of $\mathbf{T}_{S}(\mathrm{~b})$ Total regime thermal conductance for the overall structure. The gray continuous line is a fitting curve, based on (5) and (6), which best approximates the experimental points. The black line is a straight line fitting.

temperature sensor integrated in the manufactured structure. Two different control methods, namely, constant target resistance and constant heating power, were implemented. From constant target resistance measurements we observed the dependence of the thermal transients and regime on the period and duty cycle of the heating PWM signal. We demonstrated that the $\mu \mathrm{C}$ software allows us to optimize the PWM period in order to minimize unwanted temperature ripples in the normal operation of the device, which are still present if we do not choose a fast enough PWM signal heating period. By exploiting a constant power control of the heater, we were able to measure an increasing thermal

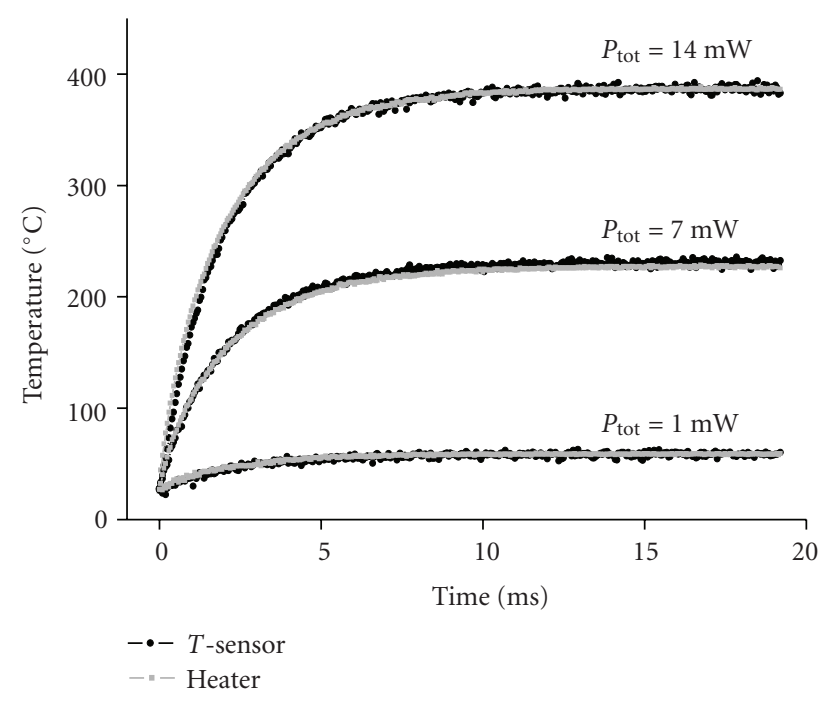

Figure 7: Heating transients of the heater and T-sensor at three different constant heating powers, from $1 \mathrm{~mW}$ to $14 \mathrm{~mW}$. Heating pulse period $\tau_{\mathrm{PWM}}=50 \mu \mathrm{s}, T_{a}=27^{\circ} \mathrm{C}$. The curves are an average of different acquisitions. We observe that the linear calibration $R_{H}=$ $R_{H}\left(T_{H}\right)$ described in Section 4.1 causes a bigger error between $T_{H}$ and $T_{T \text {-sens }}$ at intermediate values of $R_{H}$.

conductance as a function of an increasing heating power. Moreover, the thermal time constants of both the heater and the T-sensor were measured and compared in the heating phase highlighting a decreasing time constant for increasing regime temperature, as we expected from an increasing thermal conductance. Finally, we made a study over the standard deviation of the system that showed the repeatability of our measurements, highlighting a smaller standard deviation for the measurements taken on the heating resistor, compared to the $\mathrm{T}$-sensor.

In the future, these results will be useful to create a lumped-element circuit model for SPICE simulation of ULP structures. Moreover, for future work we intend to use $\tau_{\mathrm{PWM}}=20 \mu$ s to reduce the temperature ripple whatever the target heater resistance (i.e., operating temperature) could be $\left(R_{H}\right.$ between 120 and $230 \Omega$ ).

\section{References}

[1] M. Y. Afridi, J. S. Suehle, D. W. Berning et al., "A monolithic CMOS microhotplate-based gas sensor system," IEEE Sensors Journal, vol. 2, no. 6, pp. 644-655, 2002.

[2] D. Briand, B. van der Schoot, N. F. de Rooij, H. Sundgren, and I. Lundström, "Low-power micromachined MOSFET gas sensor," Journal of Microelectromechanical Systems, vol. 9, no. 3, pp. 303-308, 2000.

[3] I. Simon, N. Bârsan, M. Bauer, and U. Weimar, "Micromachined metal oxide gas sensors: opportunities to improve sensor performance," Sensors and Actuators, B, vol. 73, no. 1, pp. 1-26, 2001.

[4] I. Sayhan, A. Helwig, T. Becker et al., "Discontinuously operated metal oxide gas sensors for flexible tag microlab applications," IEEE Sensors Journal, vol. 8, no. 2, pp. 176-181, 2008. 
[5] S. Zampolli, I. Elmi, E. Cozzani et al., "Ultra-low-power components for an RFID Tag with physical and chemical sensors," Microsystem Technologies, vol. 14, no. 4-5, pp. 581588, 2008.

[6] E. Espinosa, R. Ionescu, S. Zampolli et al., "Drop-coated sensing layers on ultra low power hotplates for an RFID flexible tag microlab," Sensors and Actuators, B, vol. 144, no. 2, pp. 462-466, 2010.

[7] C.-L. Dai and M.-C. Liu, "Nanoparticle $\mathrm{SnO}_{2}$ gas sensor with circuit and microheater on chip fabricated using CMOSMEMS technique," in Proceedings of the 2nd IEEE International Conference on (NEMS '07), pp. 959-963, Bangkok, Thailand, January 2007.

[8] J. L. Merino, S. A. Bota, R. Casanova, A. Diéguez, C. Cané, and J. Samitier, "A reusable smart interface for gas sensor resistance measurement," IEEE Transactions on Instrumentation and Measurement, vol. 53, no. 4, pp. 1173-1178, 2004.

[9] A. Lombardi, M. Grassi, P. Malcovati et al., "A CMOS integrated interface circuit for metal-oxide gas sensors," Sensors and Actuators, B, vol. 142, no. 1, pp. 82-89, 2009.

[10] L. Bissi, M. Cicioni, P. Placidi, S. Zampolli, and I. Elmi, "A programmable interface circuit for an ultralow power gas sensor," IEEE Transactions on Instrumentation and Measurement, vol. PP, no. 99, pp. 1-8, 2010.

[11] P. Ruther, M. Herrscher, and O. Paul, "A micro differential thermal analysis ( $\mu \mathrm{DTA})$ system," in Proceedings of the 17th IEEE International Conference on Micro Electro Mechanical Systems (MEMS '04), pp. 165-168, Maastricht, The Netherlands, January 2004.

[12] M. Baroncini, P. Placidi, G. C. Cardinali, and A. Scorzoni, "Thermal characterization of a microheater for micromachined gas sensors," Sensors and Actuators, A, vol. 115, no. 1, pp. 8-14, 2004.

[13] S. Bicelli, A. Depari, G. Faglia et al., "Model and experimental characterization of the dynamic behavior of low-power carbon monoxide MOX sensors operated with pulsed temperature profiles," IEEE Transactions on Instrumentation and Measurement, vol. 58, no. 5, pp. 1324-1332, 2009.

[14] P. Fürjes, C. S. Dücs, M. Ádám, J. Zettner, and I. Bársony, "Thermal characterisation of micro-hotplates used in sensor structures," Superlattices and Microstructures, vol. 35, no. 3-6, pp. 455-464, 2004.

[15] J. G. A. Lira, A. C. Oliveira Jr., R. C. S. Freire, I. Doi, B. A. Luciano, and J. W. Swart, "Dynamic characterization of thermo-resistive micro-sensor," in Proceedings of the IEEE Instrumentation and Measurement Technology Conference (IMTC '05), pp. 1647-1651, Ottawa, Canada, May 2005.

[16] I. Elmi, S. Zampolli, E. Cozzani, M. Passini, G. C. Cardinali, and M. Severi, "Development of ultra low power consumption hotplates for gas sensing applications," in Proceedings of the 5th IEEE Conference on Sensors (EXCO '06), pp. 243-246, Daegu, Korea, October 2006.

[17] I. Elmi, S. Zampolli, E. Cozzani, F. Mancarella, and G. C. Cardinali, "Development of ultra-low-power consumption MOX sensors with ppb-level VOC detection capabilities for emerging applications," Sensors and Actuators, B, vol. 135, no. 1, pp. 342-351, 2008.

[18] A. Scorzoni, M. Baroncini, and P. Placidi, "On the relationship between the temperature coefficient of resistance and the thermal conductance of integrated metal resistors," Sensors and Actuators, A, vol. 116, no. 1, pp. 137-144, 2004. 

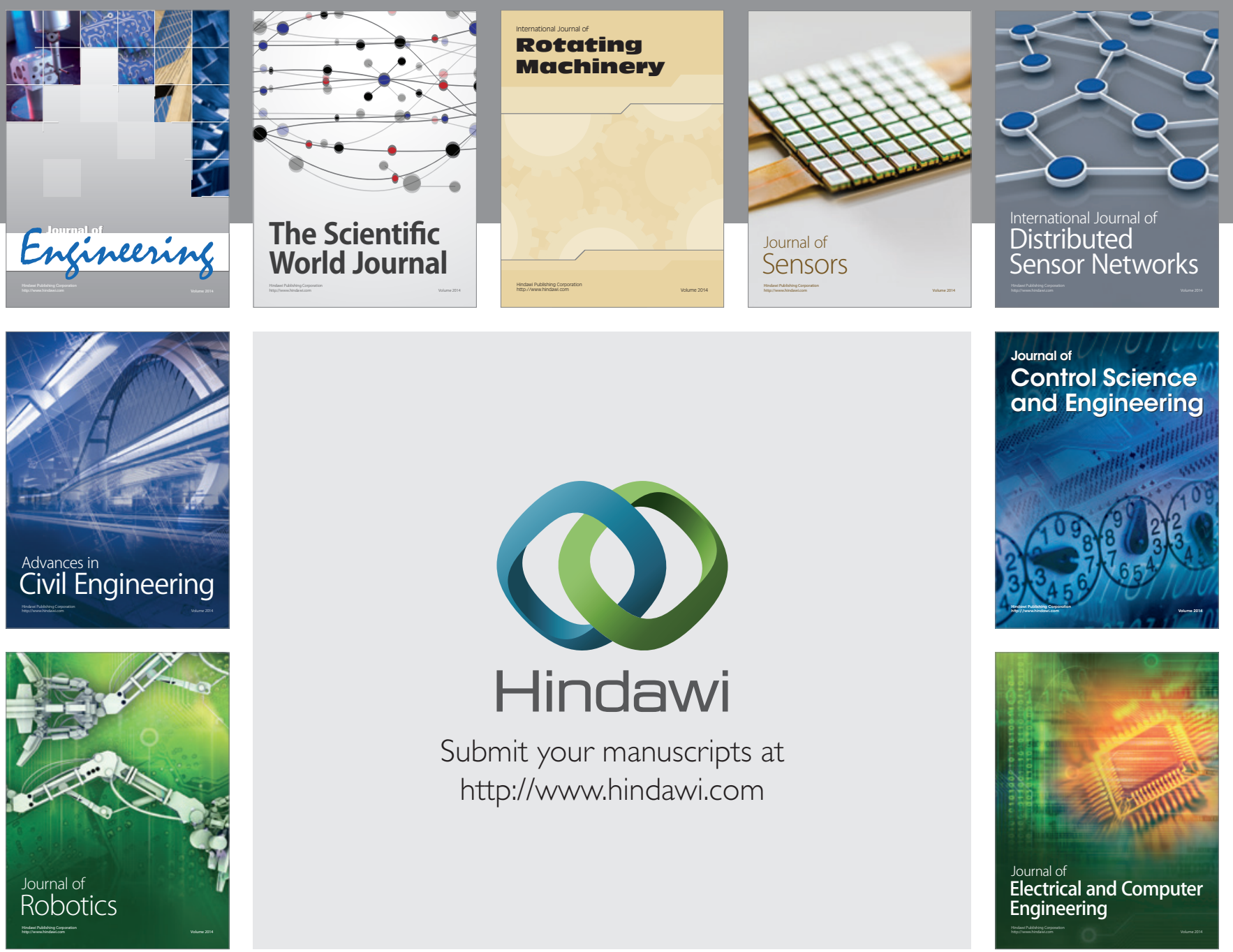

Submit your manuscripts at

http://www.hindawi.com
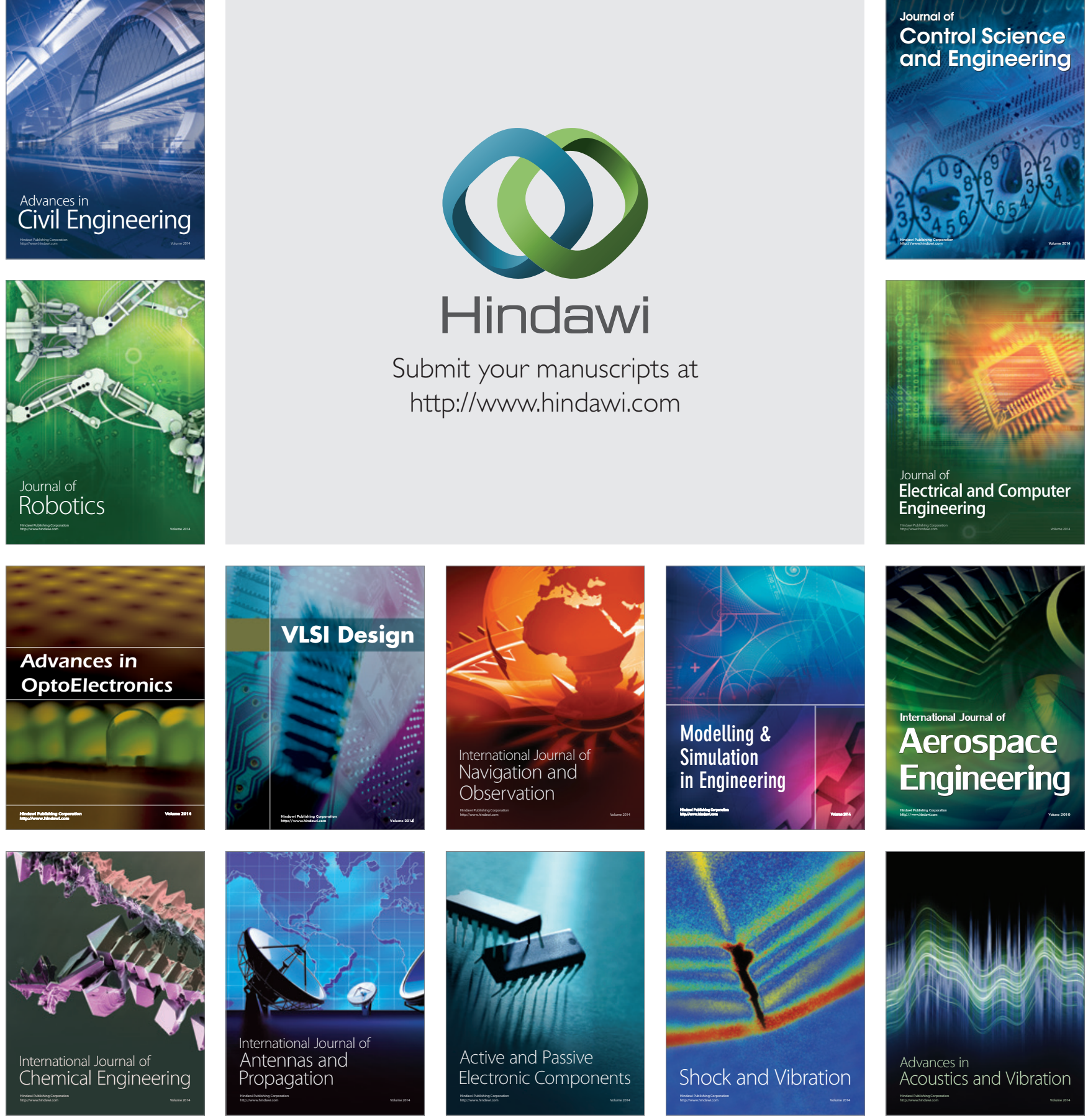